Published in final edited form as:

Clin Auton Res. 2015 August ; 25(4): 243-250. doi:10.1007/s10286-015-0301-2.

\title{
Effect of hypoxia on heart rate variability and baroreflex sensitivity during hypoglycemia in Type 1 Diabetes Mellitus
}

\author{
Jacqueline K. Limberg, Ph.D. ${ }^{1}$, Simmi Dube, M.D. ${ }^{2}$, Myrthe Kuijpers, B.S. ${ }^{1}$, Kathryn E. \\ Farni, B.S. ${ }^{1}$, Ananda Basu, M.B.B.S, M.D. ${ }^{2}$, Robert A. Rizza, M.D. ${ }^{2}$, Timothy B. Curry, M.D., \\ Ph.D. ${ }^{1}$, Rita Basu, M.D. ${ }^{2,}$, and Michael J. Joyner, M.D. ${ }^{1,{ }^{*}}$ \\ ${ }^{1}$ Department of Anesthesiology, Mayo Clinic, Rochester, MN, USA \\ ${ }^{2}$ Department of Endocrinology, Mayo Clinic, Rochester, MN
}

\begin{abstract}
Purpose-Patients with type 1 diabetes mellitus exhibit impairments in autonomic and cardiovascular control which are worsened with acute hypoglycemia-thus increasing the risk of adverse cardiovascular events. Hypoxia, as seen with the common comorbidity of sleep apnea, may lead to further autonomic dysfunction and an increased risk of ventricular arrhythmias. Therefore, we hypothesized heart rate variability (HRV) and baroreflex sensitivity (BRS) would be reduced during hypoglycemia in adults with type 1 diabetes, with a further decline when combined with hypoxia.
\end{abstract}

Methods-Subjects with type 1 diabetes $(\mathrm{n}=13$; HbA1c $=7.5 \pm 0.3 \%$, Duration of diabetes $=17 \pm 5$ yrs) completed two 180-minute hyperinsulinemic ( $2 \mathrm{mU} / \mathrm{kg}$ TBW/min), hypoglycemic ( 3.3 $\mu \mathrm{mol} / \mathrm{mL})$ clamps separated by a minimum of 1 week and randomized to normoxia $\left(\mathrm{S}_{\mathrm{p}} \mathrm{O}_{2} \sim 98 \%\right)$ or hypoxia $\left(\mathrm{S}_{\mathrm{p}} \mathrm{O}_{2} \sim 85 \%\right)$. Heart rate (electrocardiogram) and blood pressure (finger photoplethysmography) were analyzed at baseline and during the hypoglycemic clamp for measures of HRV and spontaneous cardiac BRS (sCBRS).

Results-Hypoglycemia resulted in significant reductions in HRV and SCBRS when compared with baseline levels (Main effect of hypoglycemia: $\mathrm{p}<0.05$ ). HRV and sCBRS were further impaired during hypoxia (Main effect of hypoxia: $p<0.05$ ).

Conclusions-Acute hypoxia worsens hypoglycemia-mediated impairments in autonomic and cardiovascular control in patients with type 1 diabetes, and may increase the risk of cardiovascular mortality. These results highlight the potential cumulative dangers of hypoglycemia and hypoxia in this vulnerable population.

\section{Keywords}

baroreflex sensitivity; heart rate variability; type 1 diabetes mellitus; hypoxia; hypoglycemia

Corresponding Author: Jacqueline K. Limberg, $2001^{\text {st }}$ St SW, Rochester, MN 55901, Phone: 507-255-8380, Fax: 507-255-7300, limberg.jacqueline@mayo.edu.

Denotes co-senior authorship.

CONFLICT OF INTEREST

There are no relevant conflicts of interest to disclose. 


\section{INTRODUCTION}

Patients with type 1 diabetes mellitus exhibit impairments in autonomic and cardiovascular control, including reduced baroreflex sensitivity (BRS) and heart rate variability (HRV) [16]. An attenuation of HRV-indicative of reduced vagal protection-is associated with sudden arrhythmic death and increased risk of mortality [14]. These reductions in autonomic and cardiovascular control in patients with type 1 diabetes mellitus are worsened with acute hypoglycemia [16]. Based on recent reports showing the majority of patients with type 1 diabetes experience multiple hypoglycemic events each week [22], patients with type 1 diabetes are at increased risk of hypoglycemia-induced cardiac arrhythmias and cardiovascular events $[3,27]$.

Approximately 55\% of severe hypoglycemic episodes in patients with type 1 diabetes mellitus occur during sleep [11] and almost half of adults with type 1 diabetes exhibit sleepdisordered breathing [18]. These data support the presence of regular hypoglycemic and hypoxic events in patients with type 1 diabetes. Along these lines, exposure to hyperoxia can improve measures of BRS and HRV in patients with type 1 diabetes [4, 29]. Despite these indirect observations, the combined effect of hypoxia and hypoglycemia on measures of autonomic and cardiovascular control in patients with type 1 diabetes was previously unexamined. With these ideas in mind, we hypothesized HRV and BRS would be reduced during steady-state hypoglycemia in patients with type 1 diabetes, with a further decline when combined with steady-state hypoxia.

\section{METHODS}

Methods and data regarding BRS during normoxic hypoglycemia in patients with type 1 diabetes and related experimental methods were published previously [16].

\section{Ethical approval}

The Institutional Review Board at the Mayo Clinic approved all experiments and procedures and studies were performed in accordance with the ethical standards as laid down in the 1964 Declaration of Helsinki and its later amendments. Written informed consent was obtained from all subjects prior to enrollment.

\section{Subjects}

All subjects were diagnosed with type 1 diabetes (duration of diabetes $>2$ years), were nonsmokers, and non-pregnant/breast feeding. Inclusion criteria included: HbA1c $\leq 10 \%$, creatinine $\leq .5 \mathrm{mg} / \mathrm{dL}$, and insulin pump (n=9) or multiple daily insulin injection $(\mathrm{n}=4)$ therapies. Exclusion criteria included unstable diabetic retinopathy/nephropathy, a history of unstable macrovascular disease, seizure disorder, diagnosed autonomic disorder, and/or other chronic diseases. Four subjects were taking medication for hypertension (angiotensinconverting enzyme inhibitor). Research dieticians provided advice to subjects to ensure body weight was maintained for two weeks prior to the study visits. Subjects refrained from exercise, alcohol, and caffeine for at least $24 \mathrm{~h}$ prior to the study visit. 


\section{Hypoglycemic Clamps}

Subjects were admitted to the Clinical Research Unit at 1700 on the evening prior to study. A standard meal (10 kcal $/ \mathrm{kg}, 55 \%$ carbohydrate, $30 \%$ fat, $15 \%$ protein) was eaten between 1800 and 1830 and subjects fasted thereafter until the end of the study. Subjects administered their customary dose of insulin according to their schedule for the evening meal. At 2030, subjects had two intravenous catheters placed in the forearm for insulin infusion and plasma glucose monitoring. Intravenous insulin was started at 2100 and hospital protocol was followed overnight to maintain euglycemia. Those subjects on multiple daily injection regimen $(n=4)$ were provided $50 \%$ of their nighttime basal insulin dose. For those using an insulin pump $(n=9)$, the pump was discontinued prior to starting the intravenous insulin. At 0900 (T0) the following morning, the overnight variable insulin infusion was discontinued and a constant insulin infusion (Novolin ${ }^{\circledR}$, Novo Nordisk Inc., Princeton NJ) was started at a rate of $2.0 \mathrm{mU} / \mathrm{kg} \mathrm{TBW} / \mathrm{min}$ from protocol time T0 to T180 minutes (Figure 1). Exogenous glucose (50\% dextrose) was infused during similar timepoints in amounts sufficient to maintain glucose concentrations at hypoglycemic levels $(\sim 3.3 \mu \mathrm{mol} / \mathrm{mL})$.

\section{Hypoxic Procedure}

Subjects visited the lab on two occasions: 1) normoxia visit, and 2) hypoxia visit. Visits were randomized and subjects were blinded to condition. During each visit, subjects wore a mask connected to a partial rebreathe system to effectively clamp end-tidal carbon dioxide levels [2]. Subjects were on the mask from 0900 (T0) until the end of the study (1200 noon; T180) (Figure 1). On the normoxia day, subjects breathed medical air. On the hypoxia day, the level of inspired oxygen was titrated using an anesthesia gas blender to achieve an arterial oxygen saturation of $\sim 85 \%$ [a level consistent with arterial oxygen saturations observed during sleep in patients with diabetes [21, 26]].

\section{Monitoring}

Blood pressure was monitored using finger plethysmography (NexFin, BMEYE, Amsterdam, The Netherlands). Heart rate was monitored with a 5-lead electrocardiogram (ECG; Cardiocap/5, Datex- Ohmeda) and respiration was assessed via capnography (Cardiocap/5, Datex- Ohmeda). A venous catheter was introduced in a retrograde fashion into a hand vein. The hand was placed in a heated plexiglass box $\left(55^{\circ} \mathrm{C}\right)$ for sampling of arterialized venous blood. Plasma glucose was measured every 5-10 minutes at the bedside using a glucose oxidase method (Analox Instruments USA Inc., Lunenberg, Massachusetts). Additional arterialized venous blood samples were drawn during baseline euglycemia and throughout the hyperinsulinemic hypoglycemic clamp for measures of insulin. Baseline values are reported as an average of T-30, $-20,-10$, and 0 min samples and clamp values as an average of T150, 160, 170, and $180 \mathrm{~min}$ [31] (Figure 1).

\section{Spontaneous Cardiac Baroreflex Sensitivity (sCBRS)}

sCBRS was assessed from 60 minute sections of data during euglycemia [T-60 - T0 (baseline)] and steady-state hypoglycemia [T120 - T180 (clamp)] (Figure 1). Detailed methods were published previously [16]. Briefly, the distances between all R-wave peaks of 
the ECG recording were calculated and paired with the systolic pressure wave amplitude of the preceding beat. A computer software program (LabChart7, ADInstruments) selected all sequences of three or more successive heart beats in which there were concordant increases or decreases in systolic blood pressure and R-R interval. The recordings were reviewed and non-sinus beats and segments with artifacts were removed. A linear regression was manually applied to each of the sequences and only relationships with an $\mathrm{R}^{2}>0.80$ were accepted. An average regression slope was then calculated for the acceptable sequences. The slope represented the sCBRS (ms/mmHg). Responses were also evaluated by plotting the changes in systolic pressures with heart rate (beat $/ \mathrm{min} / \mathrm{mmHg}$ ) to take into consideration the mathematical constraint of the hyperbolic relationship between R-R interval and heart rate.

\section{Heart Rate Variability (HRV)}

Following standard procedures recommended by the Task Force of the European Society of Cardiology and North American Society of Pacing and Electrophysiology [8], short-term ( 5-minute) data selections from a 5-lead ECG recording were analyzed during baseline [T-30-T0] and steady-state hypoglycemia [T150-T180] under both normoxic and hypoxic conditions (Figure 1). Physiologically stable conditions were confirmed by visual checks, ensuring only stationary segments were selected for analysis. A computer program (HRV Module, LabChart7, ADInstruments) was used to assess time domains and HRV data are reported as: Mean NN Interval (time between normal cardiac cycles, reported in ms), RMSSD (root mean square of successive R-R interval differences, reported in $\mathrm{ms}$ ), and SDNN (standard deviation of normal R-R intervals, reported in ms).

\section{Data Analysis and Statistics}

All statistical analyses were completed using SigmaStat 2.03 software (Systat Software Inc; San Jose, CA, USA). Two-way repeated measures analysis of variance was performed to compare the effect of time (baseline, clamp) and condition (normoxia, hypoxia) on primary outcome variables. Distributional assumptions were assessed and non-parametric methods were used as appropriate. Statistical significance was determined $a$ priori at the $\mathrm{a}=0.05$ level. The number of subjects $(\mathrm{n}=13)$ was selected a priori by a power test equation with $\mathrm{a}=0.05$ and power $=0.80$, using differences in BRS from previously published research in patients with type 1 diabetes mellitus [32]. Furthermore, post hoc analysis showed 13 subjects provided $77 \%$ power to detect a difference in BRS. Data are reported as Mean \pm Standard Error of the Mean.

\section{RESULTS}

\section{Subjects}

13 adults with type 1 diabetes (7M/6F) participated in the current study (Table 1). On both visits, the hyperinsulinemic hypoglycemic clamp resulted in higher insulin concentrations (Normoxia: $83 \pm 14$ to $1007 \pm 125$; Hypoxia: $90 \pm 14$ to $979 \pm 90 \mathrm{pmol} / \mathrm{L}$ ) and lower glucose concentrations (Normoxia: $8.5 \pm 1.2$ to $3.4 \pm 0.1$; Hypoxia: $6.1 \pm 0.6$ to $3.3 \pm 0.1 \mu \mathrm{mol} / \mathrm{mL}$ ) as compared to baseline (Main effect of time, $\mathrm{p} \unlhd \mathbf{}(01$ ). As designed, hypoxia resulted in a significant reduction in $\mathrm{S}_{\mathrm{p}} \mathrm{O}_{2}(97 \pm 1$ to $85 \pm 1 \%$; Main effect of condition, $\mathrm{p}<0.01)$. 


\section{Hemodynamic response to hypoxic hypoglycemia}

Diastolic and mean blood pressure did not change with hypoglycemia (Main effect of time, $\mathrm{p}=0.70$ and $\mathrm{p}=0.49$, respectively). There was an increase in systolic blood pressure and heart rate with hypoglycemia (Main effect of time, $\mathrm{p}=0.05$ and $\mathrm{p}<0.01$, respectively). Responses were not altered significantly with hypoxia (Main effect of condition, $\mathrm{p}>0.05$ ). See Table 2.

\section{Spontaneous Cardiac Baroreflex Sensitivity (sCBRS)}

One subject was excluded from analysis due to inability to achieve sufficient number of sequences; therefore, data are reported from $n=12$. There was a significant reduction in sCBRS from baseline levels during steady-state hypoglycemia (Main effect of time; ms/ $\mathrm{mmHg} \mathrm{p}=0.01$; beat $/ \mathrm{min} / \mathrm{mmHg}, \mathrm{p}=0.19$ ). sCBRS was lower under hypoxic conditions when compared to normoxia (Main effect of condition; $\mathrm{ms} / \mathrm{mmHg}, \mathrm{p}=0.03$; beat $/ \mathrm{min} / \mathrm{mmHg}$, $\mathrm{p}=0.05)$. See Figure 2 .

\section{Heart Rate Variability (HRV)}

Mean NN Interval was significantly reduced during hypoglycemia (Main effect of time, $\mathrm{p}<0.01$; Interaction of time and condition, $\mathrm{p}=0.02$ ). RMSSD and SDNN were reduced during hypoglycemia, but only under hypoxic conditions (Interaction of time and condition, $\mathrm{p}=0.05$ and $\mathrm{p}=0.02$, respectively). The reduction $(\Delta)$ in Mean NN Interval and SDNN was greater during hypoxia when compared to normoxia ( $\mathrm{p}=0.01$ and $\mathrm{p}=0.02$, respectively). See Figure 3.

It is important to note, changes in HRV may be affected by changes in ventilation [8]. Changes in ventilation, however, are unlikely to influence present findings because: 1) The ventilatory response to hypoxia in patients with type 1 diabetes is blunted [7, 13, 20, 33], 2) A hypoxic ventilatory decline occurs with sustained hypoxemia [25], and 3) Measures of respiratory rate were not significantly different between baseline, normoxic hypoglycemia, and hypoxic hypoglycemia ( $\mathrm{n}=4$; Normoxia: $14 \pm 1$ to $15 \pm 2 \mathrm{breath} / \mathrm{min}$; Hypoxia: $13 \pm 1$ to $15 \pm 2$ breath/min; Effect of gas, $\mathrm{p}>0.05$; Interaction of gas and time, $\mathrm{p}>0.05$ ).

\section{DISCUSSION}

We have shown previously that impairments in autonomic and cardiovascular function in patients with type 1 diabetes are worsened with acute hypoglycemia [16]. Novel findings from the present study show hypoglycemia-mediated impairments in autonomic and cardiovascular control are further worsened under conditions of hypoxia. These data highlight the cumulative dangers of hypoglycemia and hypoxia in this vulnerable population.

\section{Impaired Autonomic Control in Type 1 Diabetes Mellitus}

Beat-to-beat variations in heart rate represent a fine-tuning of cardiovascular control mechanisms. Reduced HRV is indicative of impaired vagal control and altered sympathovagal balance, and is an independent predictor of sudden arrhythmic death and cardiovascular mortality [14]. Measures of HRV have repeatedly been shown to be impaired in patients with type 1 diabetes $[9,15,17,32,34]$ and recently our group found these 
impairments are worsened during acute hypoglycemia [16]. Impairments in HRV, and specifically vagal tone, will increase the risk of ventricular arrhythmias and cardiovascular mortality in this clinical population [3, 27]. Baroreflex sensitivity (BRS)-the ability to sense and appropriately respond to fluctuations in blood pressure-may be considered a more sensitive measure of autonomic function when compared with HRV [10] and was also impaired both during euglycemia and acute hypoglycemia in patients with type 1 diabetes [16]. Taken together, patients with type 1 diabetes exhibit large impairments autonomic and cardiovascular control during euglycemia that worsens during hypoglycemia-putting them at increased risk of cardiovascular mortality. This is especially concerning given recent reports show greater than $60 \%$ of patients with type 1 diabetes-experience one or more hypoglycemic events each week [22].

\section{Combined effect of Hypoxia and Hypoglycemia}

As mentioned above, approximately $55 \%$ of severe hypoglycemic episodes in patients with type 1 diabetes occur during sleep [11] and almost half of adults with type 1 diabetes have been shown to exhibit sleep-disordered breathing [18]. Interestingly, exposure to hyperoxia can improve measures of BRS and HRV in patients with type 1 diabetes $[4,29]$. These data support the presence of regular hypoglycemic and hypoxic events in patients with type 1 diabetes, the potential for such events to contribute to impaired autonomic and cardiovascular control, and the beneficial effects of treating such impairments with supplemental oxygen. Despite these indirect observations, the combined effect of hypoxia and hypoglycemia on measures of autonomic and cardiovascular control in patients with type 1 diabetes was previously unexamined.

Results from the present study show impairments in baseline HRV [16] are worsened during hypoxia. More specifically, we observed a reduction in RMSSD, SDNN, and Mean NN interval when hypoglycemia was combined with hypoxia $\left[\sim 85 \% \mathrm{~S}_{\mathrm{p}} \mathrm{O}_{2}\right.$ - a level consistent with arterial oxygen saturations observed during sleep in patients with diabetes [21, 26]] (Figure 3). Measures of dispersion of normal R-R intervals, such as RMSSD or SDNN, provide insight into the balance between sympathetic and parasympathetic branches of the autonomic nervous system, such that a reduction in RMSSD may be indicative of a reduction in parasympathetic activity and vagal control of heart rate [8]. Further, a fall in SDNN and Mean NN Interval are consistent with impaired sympathetic and parasympathetic modulation [8], and highlight the potential risk of combined hypoglycemia and hypoxia on normal cardiac function.

In addition to impairments in HRV, hypoglycemia-mediated impairments scBRS were further worsened under conditions of hypoxia (Figure 2). Activation of the carotid chemoreceptors is known to reduce baroreflex sensitivity and/or shift the baroreflex stimulus-response curve $[6,12,19]$. Given both hypoglycemia and hypoxia may act at the level of the carotid chemoreceptors to alter afferent activity [23], it is reasonable to propose improving chronic activity of the carotid chemoreceptors in patients with type 1 diabetes either through improved glycemic control, a reduction in the number of apneic events, and/or treatment for chronic hypoxemia-might lower the risk of ventricular arrhythmias and cardiovascular mortality. 
The observed reductions in measures of cardiac and autonomic control may be the result of chronic sympathoadrenal dysfunction and the inability to appropriately respond to changes in systemic glucose concentrations in type 1 diabetes. In addition to chronic impairments in sympathoadrenal function with type 1 diabetes, both hypoxia and hypoglycemia contribute to a reduction in metabolic substrate and metabolic depression of neuronal function. Along these lines, hypoglycemia results in a reduction in nerve action potential and velocity that is more pronounced in nerves exposed to hypoxia [28]. In addition, diabetes increases the potential for loss of synaptic function and axonal conduction when hypoxia is combined with hypoglycemia [30]. Such impairments likely contribute to the loss of integrity of motor descending pathways and/or the transfer of afferent information from peripheral sensors.

\section{Experimental Considerations}

Although much of the rationale for the present study was based on the high prevalence of both hypoglycemic events and sleep disordered breathing in patients with type 1 diabetes, it is important to note there are a number of differences between repeated acute hypoxic challenges (e.g. apneic events) and more long-term exposures (e.g. 3 hours of experimental hypoxia), in addition to acute and more prolonged hypoglycemic events. Given this is the first study of its kind examining combined hypoglycemia and hypoxia, it was important to first assess responses to changes in the steady-state. However, future studies should consider effects of intermittent exposures, in addition to the potential for circadian changes in glucose levels and chemoreceptor activation, and the impact of prolonged chemoreceptor excitation following nocturnal apneic events. It is also important to note 4 of the 13 subjects ( $30 \%)$ were taking blood pressure medications. Importantly, significant differences in main outcome variables between medicated and non-mediated individuals were not observed. Furthermore, $\sim 30 \%$ of patients with type 1 diabetes are on anti-hypertensive medications [1], thus the inclusion of such patient groups is clinically relevant.

\section{Clinical Implications}

Intensive glycemic control increases the risk of hypoglycemia and thus the risk of ventricular arrhythmias and cardiovascular mortality in patients with type 1 diabetes [3, 27]. Additionally, the effect of hypoglycemia on cardiac dysfunction is worsened in patients exhibiting additional cardiovascular disease risk factors (sleep apnea, chronic respiratory disease, etc) [5]. Our results suggest some of the observed cardiovascular risk in patients with diabetes may be the result of the combined effects of hypoglycemia and hypoxia on cardiac autonomic function. Although the present study did not include healthy controls, recent data suggests arrhythmic events that may be well tolerated by young healthy individuals could be clinically relevant in patients with diabetes [5]. Consistent with this idea, type 1 diabetes is also associated with an increased risk of sudden cardiac death [3] and present data support the idea that the combined effect of hypoglycemia and hypoxia may also contribute to cardiorespiratory failure and sudden nocturnal cardiac death [24].

\section{CONCLUSIONS}

When hypoglycemia is combined with systemic hypoxia, any hypoglycemia-mediated impairment in measures of cardiovascular and autonomic function is worsened in adults 
with type 1 diabetes. These results highlight the cumulative dangers of poorly controlled diabetes and chronic hypoxia, and the importance of therapies directed toward improving glycemic control, reducing apneic events, and/or treating chronic hypoxemia in this clinical population.

\section{Acknowledgments}

The authors wish to acknowledge the contributions of the nursing and technical staff: Cheryl Shonkwiler, Barbara Norby, Shelly Roberts, Karen Krucker, Sarah Wolhart, Jean Knutson, Brent McConahey, Pamela Reich, Nancy Meyer, Pam Engrav, Christopher Johnson, and Kate Malterer of the Mayo Clinic. In addition, we thank the Clinical Research Unit staff at Mayo Clinic, the Immunochemical Core Laboratory at Mayo Clinic, in particular Hilary Blair. We are deeply indebted to our research participants. Thanks to Brandon Bucher and Brenton Nelson at AD Instruments for the development of the Spontaneous Cardiac Baroreflex Analysis Program. Funding sources: NIH DK090541 (MJJ, RB), NIH NS32352 (MJJ), NIH T32 DK07352 (JKL), NIH F32 HL120570 (JKL), NIH 1 UL1 RR024150 (Mayo Clinic CTSA, MJJ), NIH DK29953 (RB).

\section{References}

1. Arauz-Pacheco C, Parrott MA, Raskin P. The treatment of hypertension in adult patients with diabetes. Diabetes Care. 2002; 25:134-147. [PubMed: 11772914]

2. Banzett RB, Garcia RT, Moosavi SH. Simple contrivance "clamps" end-tidal PCO(2) and PO(2) despite rapid changes in ventilation. J Appl Physiol. 2000; 88:1597-1600. [PubMed: 10797118]

3. Bergner DW, Goldberger JJ. Diabetes mellitus and sudden cardiac death: what are the data? Cardiol J. 2010; 17:117-129. [PubMed: 20544609]

4. Bernardi L, Rosengard-Barlund M, Sandelin A, Makinen VP, Forsblom C, Groop PH. Short-term oxygen administration restores blunted baroreflex sensitivity in patients with type 1 diabetes. Diabetologia. 2011; 54:2164-2173. [PubMed: 21647699]

5. Chow E, Bernjak A, Williams S, Fawdry RA, Hibbert S, Freeman J, Sheridan PJ, Heller SR. Risk of cardiac arrhythmias during hypoglycemia in patients with type 2 diabetes and cardiovascular risk. Diabetes. 2014; 63:1738-1747. [PubMed: 24757202]

6. Cooper VL, Pearson SB, Bowker CM, Elliott MW, Hainsworth R. Interaction of chemoreceptor and baroreceptor reflexes by hypoxia and hypercapnia - a mechanism for promoting hypertension in obstructive sleep apnoea. J Physiol. 2005; 568:677-687. [PubMed: 16109727]

7. Duennwald T, Bernardi L, Gordin D, Sandelin A, Syreeni A, Fogarty C, Kyto JP, Gatterer H, Lehto M, Horkko S, Forsblom C, Burtscher M, Groop PH. Effects of a single bout of interval hypoxia on cardiorespiratory control in patients with type 1 diabetes. Diabetes. 2013; 62:4220-4227. [PubMed: 23733200]

8. Electrophysiology TFotESoCatNASoPa . Heart rate variability: standards of measurement, physiological interpretation and clinical use. Circulation. 1996; 93:1043-1065. [PubMed: 8598068]

9. Ewing DJ, Neilson JM, Shapiro CM, Stewart JA, Reid W. Twenty four hour heart rate variability: effects of posture, sleep, and time of day in healthy controls and comparison with bedside tests of autonomic function in diabetic patients. Br Heart J. 1991; 65:239-244. [PubMed: 2039667]

10. Frattola A, Parati G, Gamba P, Paleari F, Mauri G, Di Rienzo M, Castiglioni P, Mancia G. Time and frequency domain estimates of spontaneous baroreflex sensitivity provide early detection of autonomic dysfunction in diabetes mellitus. Diabetologia. 1997; 40:1470-1475. [PubMed: 9447956]

11. Group TDR. Epidemiology of severe hypoglycemia in the diabetes control and complications trial. Am J Med. 1991; 90:450-459. [PubMed: 2012085]

12. Halliwill JR, Morgan BJ, Charkoudian N. Peripheral chemoreflex and baroreflex interactions in cardiovascular regulation in humans. J Physiol. 2003; 552:295-302. [PubMed: 12897165]

13. Homma I, Kageyama S, Nagai T, Taniguchi I, Sakai T, Abe M. Chemosensitivity in patients with diabetic neuropathy. Clin Sci (Lond). 1981; 61:599-603. [PubMed: 6793279] 
14. Kleiger RE, Miller JP, Bigger JT Jr, Moss AJ. Decreased heart rate variability and its association with increased mortality after acute myocardial infarction. Am J Cardiol. 1987; 59:256-262. [PubMed: 3812275]

15. Koivikko ML, Salmela PI, Airaksinen KE, Tapanainen JS, Ruokonen A, Makikallio TH, Huikuri HV. Effects of sustained insulin-induced hypoglycemia on cardiovascular autonomic regulation in type 1 diabetes. Diabetes. 2005; 54:744-750. [PubMed: 15734851]

16. Limberg JK, Farni KE, Taylor JL, Dube S, Basu A, Basu R, Wehrwein EA, Joyner MJ. Autonomic control during acute hypoglycemia in type 1 diabetes mellitus. Clin Auton Res. 2014

17. Malpas SC, Maling TJ. Heart-rate variability and cardiac autonomic function in diabetes. Diabetes. 1990; 39:1177-1181. [PubMed: 2210071]

18. Manin G, Pons A, Baltzinger P, Moreau F, Iamandi C, Wilhelm JM, Lenoble P, Kessler L, Kessler R. Obstructive sleep apnoea in people with Type 1 diabetes: prevalence and association with micro- and macrovascular complications. Diabet Med. 2014

19. Monahan KD, Leuenberger UA, Ray CA. Effect of repetitive hypoxic apnoeas on baroreflex function in humans. J Physiol. 2006; 574:605-613. [PubMed: 16709638]

20. Montserrat JM, Cochrane GM, Wolf C, Picado C, Roca J, Agusti Vidal A. Ventilatory control in diabetes mellitus. Eur J Respir Dis. 1985; 67:112-117. [PubMed: 4054261]

21. Neumann C, Martinez D, Schmid H. Nocturnal oxygen desaturation in diabetic patients with severe autonomic neuropathy. Diabetes Res Clin Pract. 1995; 28:97-102. [PubMed: 7587925]

22. Ostenson CG, Geelhoed-Duijvestijn P, Lahtela J, Weitgasser R, Markert Jensen M, PedersenBjergaard U. Self-reported non-severe hypoglycaemic events in Europe. Diabet Med. 2014; 31:92-101. [PubMed: 23796113]

23. Pardal R, Lopez-Barneo J. Low glucose-sensing cells in the carotid body. Nat Neurosci. 2002; 5:197-198. [PubMed: 11850631]

24. Parekh B. The mechanism of dead-in-bed syndrome and other sudden unexplained nocturnal deaths. Curr Diabetes Rev. 2009; 5:210-215. [PubMed: 19604134]

25. Powell FL, Milsom WK, Mitchell GS. Time domains of the hypoxic ventilatory response. Respir Physiol. 1998; 112:123-134. [PubMed: 9716296]

26. Resnick HE, Redline S, Shahar E, Gilpin A, Newman A, Walter R, Ewy GA, Howard BV, Punjabi NM. Diabetes and sleep disturbances: findings from the Sleep Heart Health Study. Diabetes Care. 2003; 26:702-709. [PubMed: 12610025]

27. Schonauer M, Thomas A, Morbach S, Niebauer J, Schonauer U, Thiele H. Cardiac autonomic diabetic neuropathy. Diab Vasc Dis Res. 2008; 5:336-344. [PubMed: 18958844]

28. Stecker MM, Stevenson M. Effect of glucose concentration on peripheral nerve and its response to anoxia. Muscle Nerve. 2014; 49:370-377. [PubMed: 23733393]

29. Sun TB, Yang CC, Kuo TB. Effect of hyperbaric oxygen on cardiac neural regulation in diabetic individuals with foot complications. Diabet Med. 2006; 23:360-366. [PubMed: 16620263]

30. Tekkok SB, Godfraind JM, Krnjevic K. Moderate hypoglycemia aggravates effects of hypoxia in hippocampal slices from diabetic rats. Neuroscience. 2002; 113:11-21. [PubMed: 12123680]

31. Wehrwein EA, Curry TB, Basu A, Rizza RA, Basu R, Joyner MJ. Do the carotid bodies modulate hypoglycemic counterregulation and baroreflex control of blood pressure in humans? Adv Exp Med Biol. 2012; 758:129-135. [PubMed: 23080153]

32. Weston PJ, Panerai RB, McCullough A, McNally PG, James MA, Potter JF, Thurston H, Swales JD. Assessment of baroreceptor-cardiac reflex sensitivity using time domain analysis in patients with IDDM and the relation to left ventricular mass index. Diabetologia. 1996; 39:1385-1391. [PubMed: 8933009]

33. Williams JG, Morris AI, Hayter RC, Ogilvie CM. Respiratory responses of diabetics to hypoxia, hypercapnia, and exercise. Thorax. 1984; 39:529-534. [PubMed: 6431627]

34. Ziegler D, Laude D, Akila F, Elghozi JL. Time- and frequency-domain estimation of early diabetic cardiovascular autonomic neuropathy. Clin Auton Res. 2001; 11:369-376. [PubMed: 11794718] 


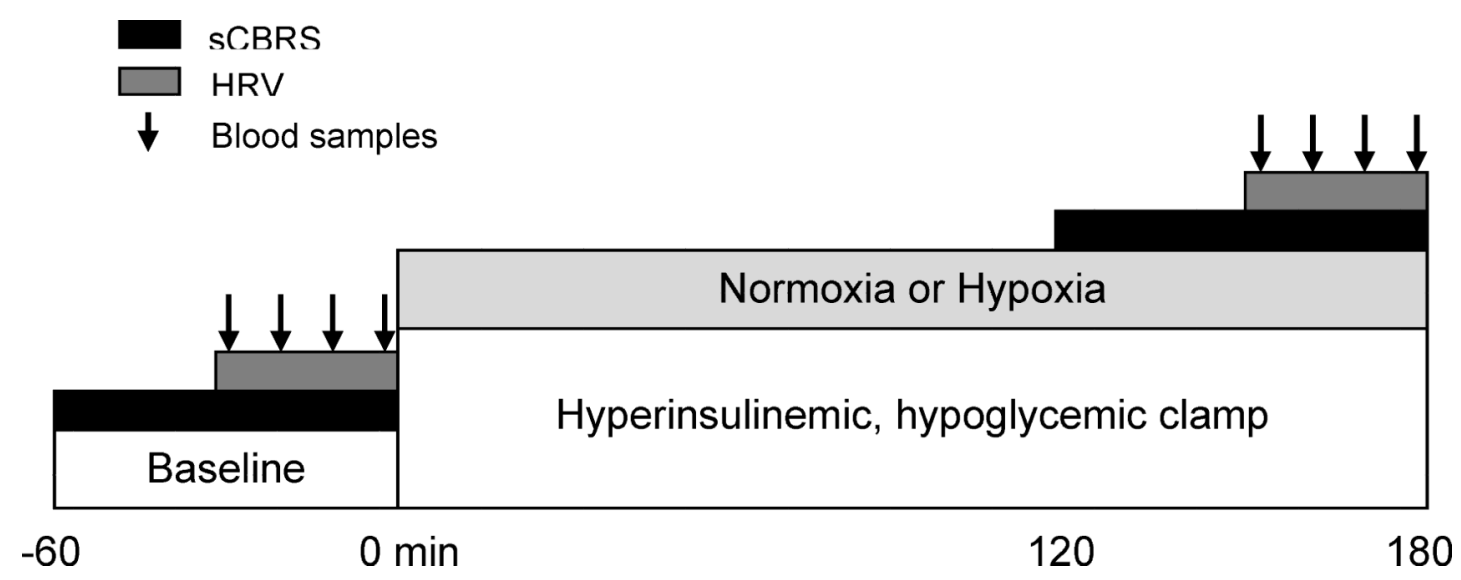

Figure 1. Study timeline

Black bars show 60 minute sections of data during euglycemia [T-60 - T0 (baseline)] and steady-state hypoglycemia [T120 - T180 (clamp)] used for measures of sCBRS. Grey bars show sections of data used for measures of HRV. Arrows show timing of blood draws. Baseline blood samples are reported as an average of T-30, -20, -10, and 0 min and clamp values as an average of T150, 160, 170, and $180 \mathrm{~min}$ blood samples. 
A

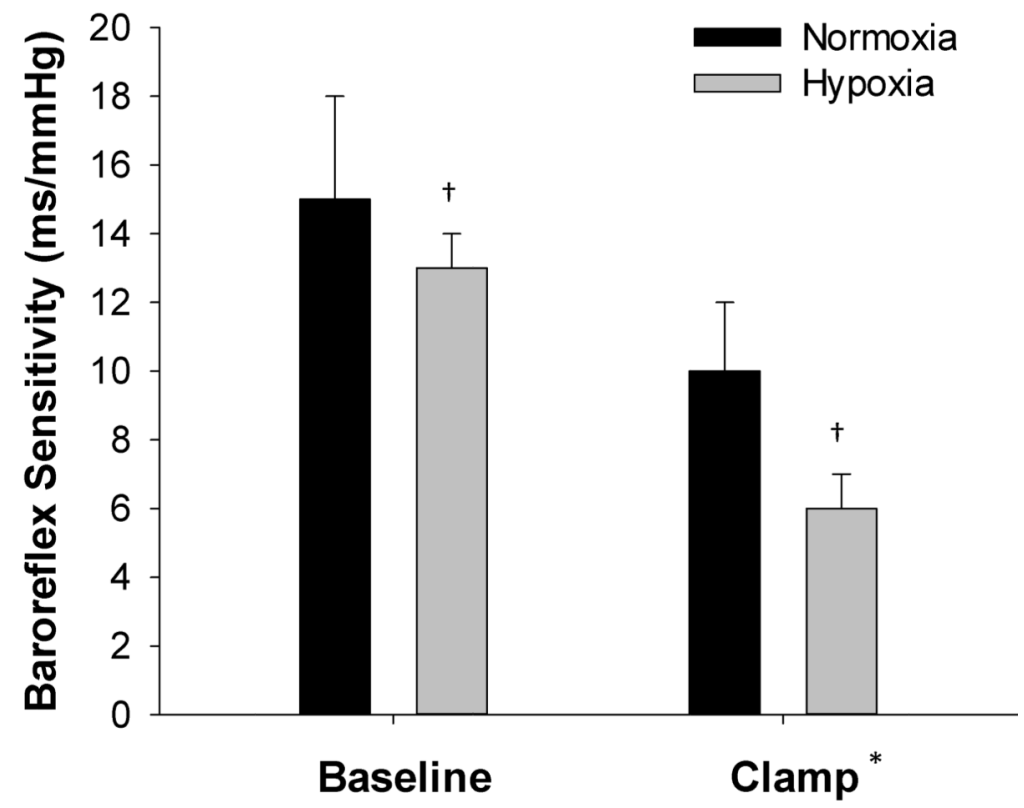

B

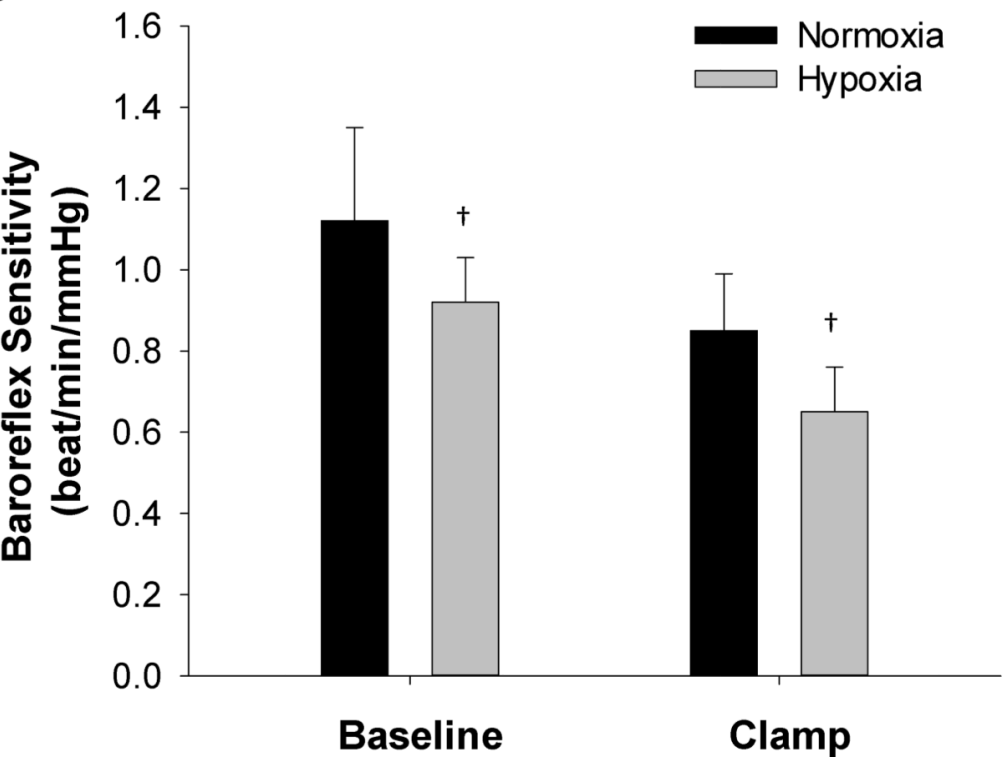

Figure 2. Changes in measures of baroreflex sensitivity $(n=12)$. There was a significant reduction in sCBRS from baseline levels during steady-state hypoglycemia $(* \mathrm{p}<0.05$ vs baseline). sCBRS was lower under hypoxic conditions when compared to normoxia ( $† \mathrm{p} \unlhd 0.05$ vs Normoxia). 
A

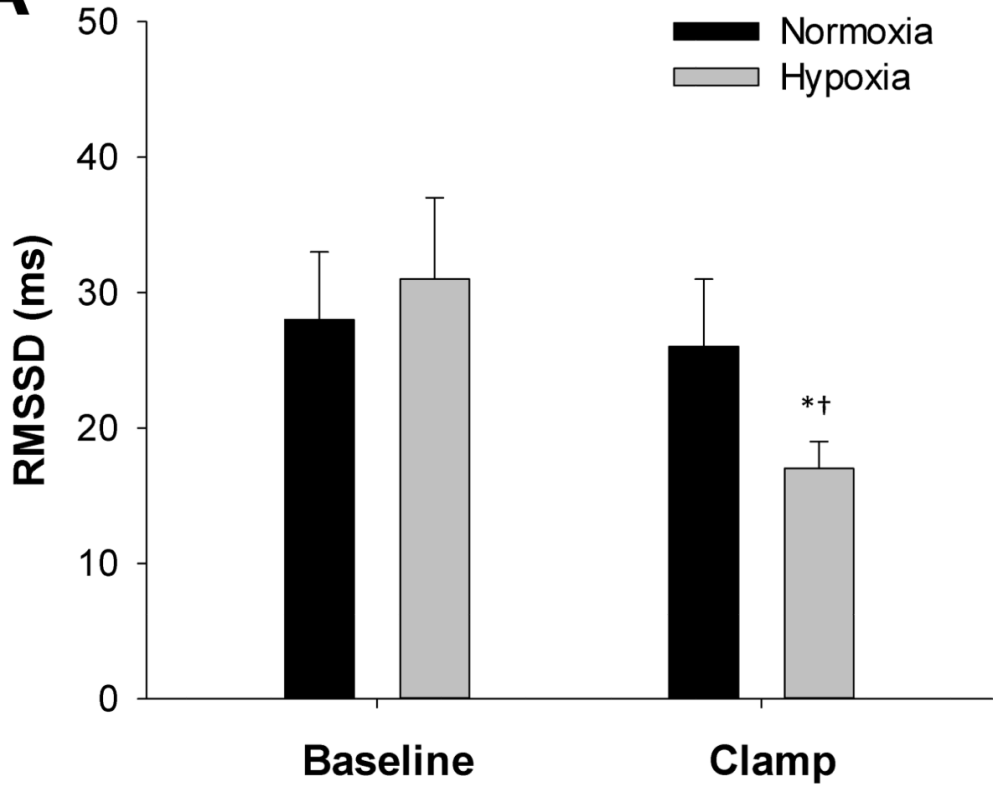

B

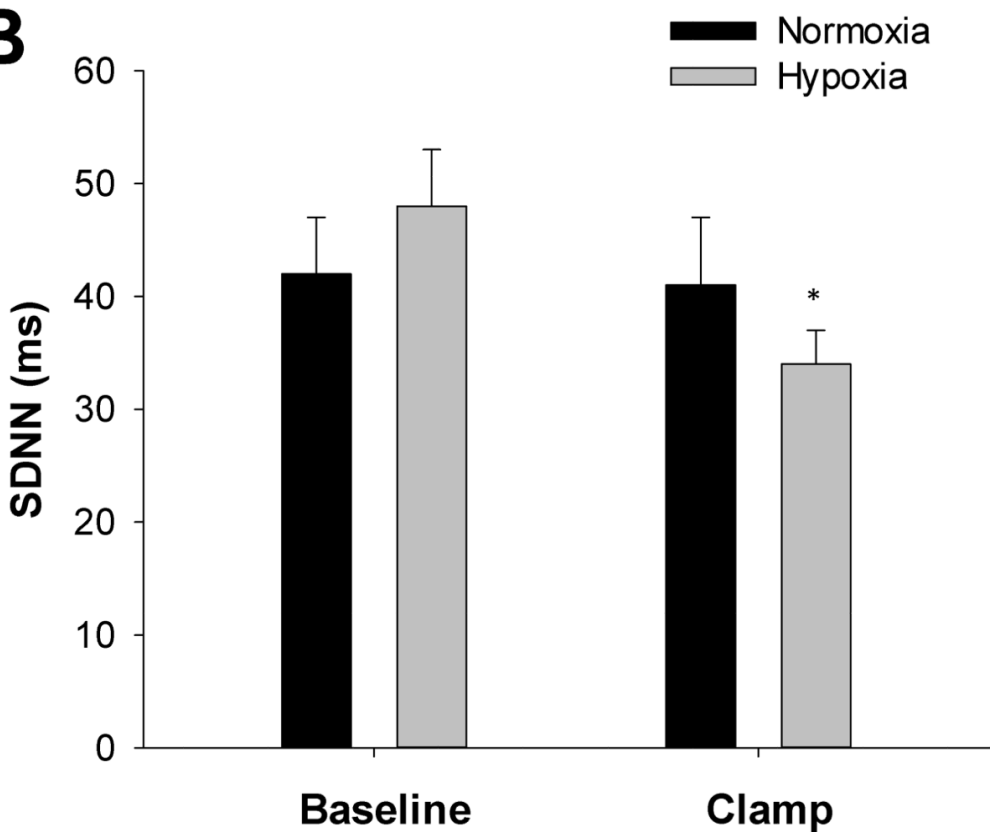


C

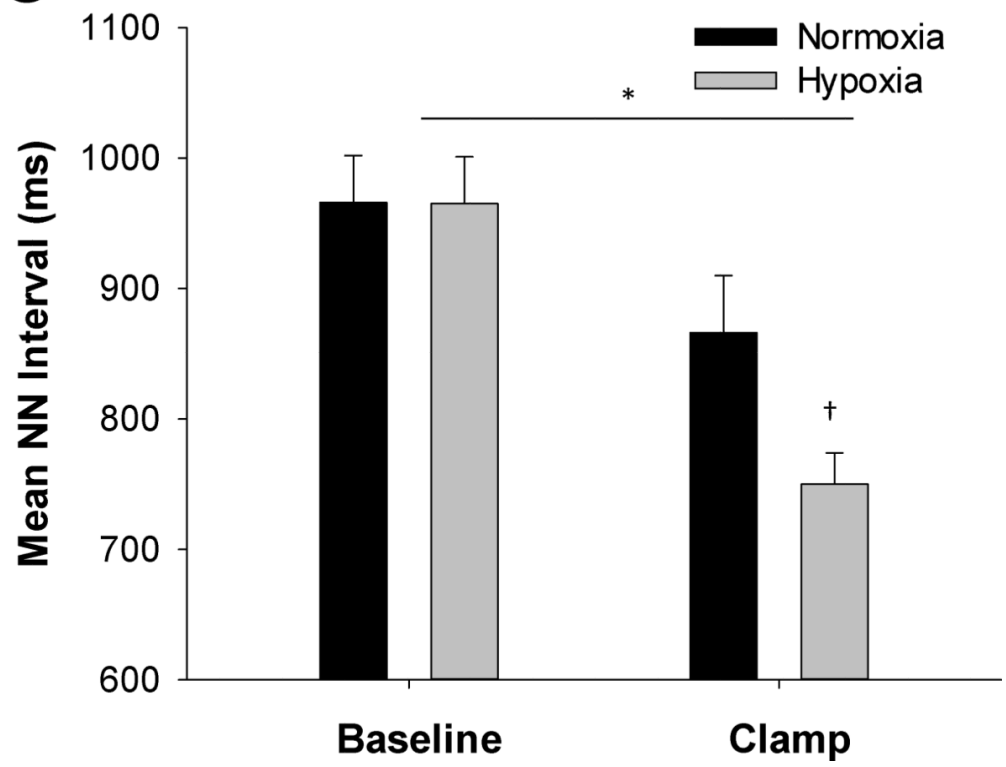

Figure 3. Changes in measures of heart rate variability $(n=13)$. RMSSD and SDNN were reduced during hypoglycemia, but only under hypoxic conditions (*p $₫ .05$ vs baseline). Mean NN Interval was significantly reduced during hypoglycemia (*p $₫ .05$ vs baseline). Absolute measures of mean NN Interval and RMSSD during the clamp were lower during hypoxia when compared to normoxia ( $\dagger \mathrm{p} \unlhd .05$ vs Normoxia). 


\section{Table 1}

\section{Subject Demographics}

Demographic data from patients with type 1 diabetes were published previously [15].

\begin{tabular}{lc}
\hline Characteristics & T1DM \\
\hline Sex $(\mathrm{M} / \mathrm{F})$ & $7 / 6$ \\
Age (years) & $43 \pm 12$ \\
Height $(\mathrm{cm})$ & $172 \pm 3$ \\
Weight $(\mathrm{kg})$ & $76 \pm 5$ \\
BMI $\left(\mathrm{kg} / \mathrm{m}^{2}\right)$ & $26 \pm 2$ \\
HbA1c $(\%)$ & $7.5 \pm 0.3$ \\
Duration of Diabetes $(\mathrm{yr})$ & $17 \pm 5$ \\
\hline
\end{tabular}


Table 2

\section{Changes in catecholamines and hemodynamic variables}

Data during normoxic hypoglycemia in patients with type 1 diabetes were published previously [15]

\begin{tabular}{ccc}
\hline & Baseline & Clamp \\
\hline \multicolumn{4}{c}{ Systolic Blood Pressure } & $(\mathbf{m m H g})$ \\
Normoxia & $123 \pm 4$ & $127 \pm 4^{*}$ \\
Hypoxia & $123 \pm 4$ & $133 \pm 4^{*}$ \\
Diastolic Blood Pressure & $(\mathbf{m m H g})$ \\
Normoxia & $67 \pm 2$ & $65 \pm 2$ \\
Hypoxia & $64 \pm 3$ & $68 \pm 3$ \\
Mean Blood Pressure (mmHg) \\
Normoxia & $87 \pm 3$ & $86 \pm 2$ \\
Hypoxia & $86 \pm 3$ & $90 \pm 3$ \\
Heart Rate (beat/min) & \\
Normoxia & $63 \pm 2$ & $73 \pm 3 *$ \\
Hypoxia & $64 \pm 2$ & $80 \pm 2 *$ \\
\hline
\end{tabular}

Mean \pm SE ( $n=13)$. Main effect of clamp ( ${ }^{*} p<0.05$ vs Baseline). Main effect of gas ( $\dagger p<0.05$ vs Normoxia). 